\title{
In-situ small-scale mechanical testing of fast reactor mixed oxide pins: completion report
}

Fabiola Cappia, Brandon D Miller, Daniel J Murray, Alexander J Winston, Jason M Harp, David Frazer

January 2019

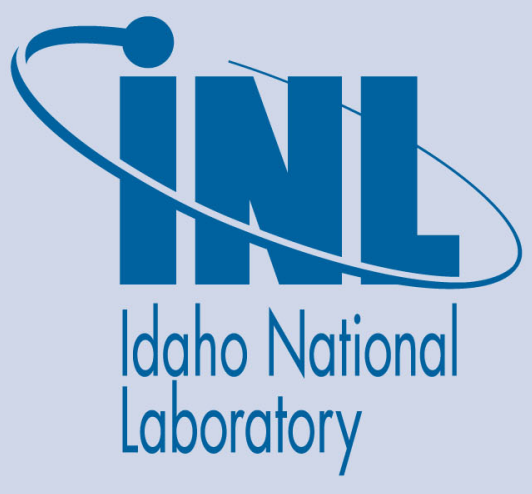

The INL is a U.S. Department of Energy National Laboratory operated by Battelle Energy Alliance 


\section{In-situ small-scale mechanical testing of fast reactor mixed oxide pins: completion report}

Fabiola Cappia, Brandon D Miller, Daniel J Murray, Alexander J Winston, Jason M Harp, David Frazer

January 2019

Idaho National Laboratory Idaho Falls, Idaho 83415

http://www.inl.gov

Prepared for the

U.S. Department of Energy

Under DOE Idaho Operations Office

Contract 18-1452 


\title{
In-situ small-scale mechanical testing of fast reactor mixed oxide pins: completion report
}

\author{
F. Cappia, D. Frazer, B. Miller, D. Murray, A. Winston and J. Harp
}

Date of completion: 01/28/2019

Sample ID and current location of sample: MNT69B-1, EML

\section{Introduction}

Throughout its life in the reactor, the nuclear fuel is subjected to a variety of phenomena that clearly impact the mechanical properties of the material. First, nucleation and growth of fission gas bubbles decreases the load-bearing capabilities of the fuel matrix. Secondly, accumulation of solid fission products either in solution in the matrix or in forms of segregated precipitates as well as irradiation damage alter the chemical bonding in the fuel [1]. These processes continue to a less extent even after discharge from the reactor, due to the internal decay of the produced fission products [2].

Knowledge of fuel mechanical properties, e.g., the elastic moduli, is crucial to establish models that can accurately predict the mechanical response of fuel pins [3]. This is particularly true for hard and brittle materials such as oxide nuclear fuels which can imposes high contact pressure on the cladding due to limited deformability of ceramics fuels at low operating temperatures. However, the intrinsic nature of irradiated fuel poses serious challenges to the measurements of its mechanical properties. Due to the high thermal gradients established during irradiation, fracture of the fuel pellets occurs. In addition, further cracking might evolve during cooling of the pins. Therefore, conventional macro-mechanical tests such as compression tests cannot be applied and won't capture the radial heterogeneity of the structure. Moreover, the high radioactivity of the spent fuel strongly limits sample handling capabilities.

Scaling of mechanical testing into the micro and submicron range has been long pursued in the materials science community. The most famous example is the development of dynamic nanoindentation techniques as well as in-situ micromechanical testing. These new capabilities are particularly attractive to study the heterogeneous structure of the irradiated fuels. Small scale mechanical testing of single features such as grain boundaries or precipitates allows tackling the materials heterogeneities arising from irradiation. Another main advantage is the reduction of radioactive material necessary for testing which reduces the dose to workers.

The micro-scale mechanical testing has been recently extended to nuclear fuels. A few results have been published on irradiated ceramics oxides by different research groups [3-7]. Most of the work has been conducted on irradiated $\mathrm{UO}_{2}$ containing various additives. Some results on Light Water Reactor (LWR) mixed oxide (MOX) were obtained too [7]. So far, no published data are available on Sodium Fast Reactor (SFR) MOX and not above $120 \mathrm{GWd} / \mathrm{tHM}$. In this project, by combining the newly developed capabilities of fast milling micrometer-size range samples with the shielded Plasma Focused lon Beam (PFIB) with the in-situ instrumentation for micro-mechanical testing at Idaho National Laboratory (INL), we explore an experimental protocol to measure mechanical properties of highly radioactive fuels with 
high Pu content while following the principle of ALARA (As Low As Reasonably Achievable).

\section{Materials and experimental approach}

The sample belongs to a MOX legacy pin available in the hot cell at the Hot Cell Examination Facility (HFEF) of INL. The pin was irradiated in the Fast Flux Test Facility (FFTF) between 1985 and 1992. Asfabricated fuel characteristics are summarized in Table 1 [8].

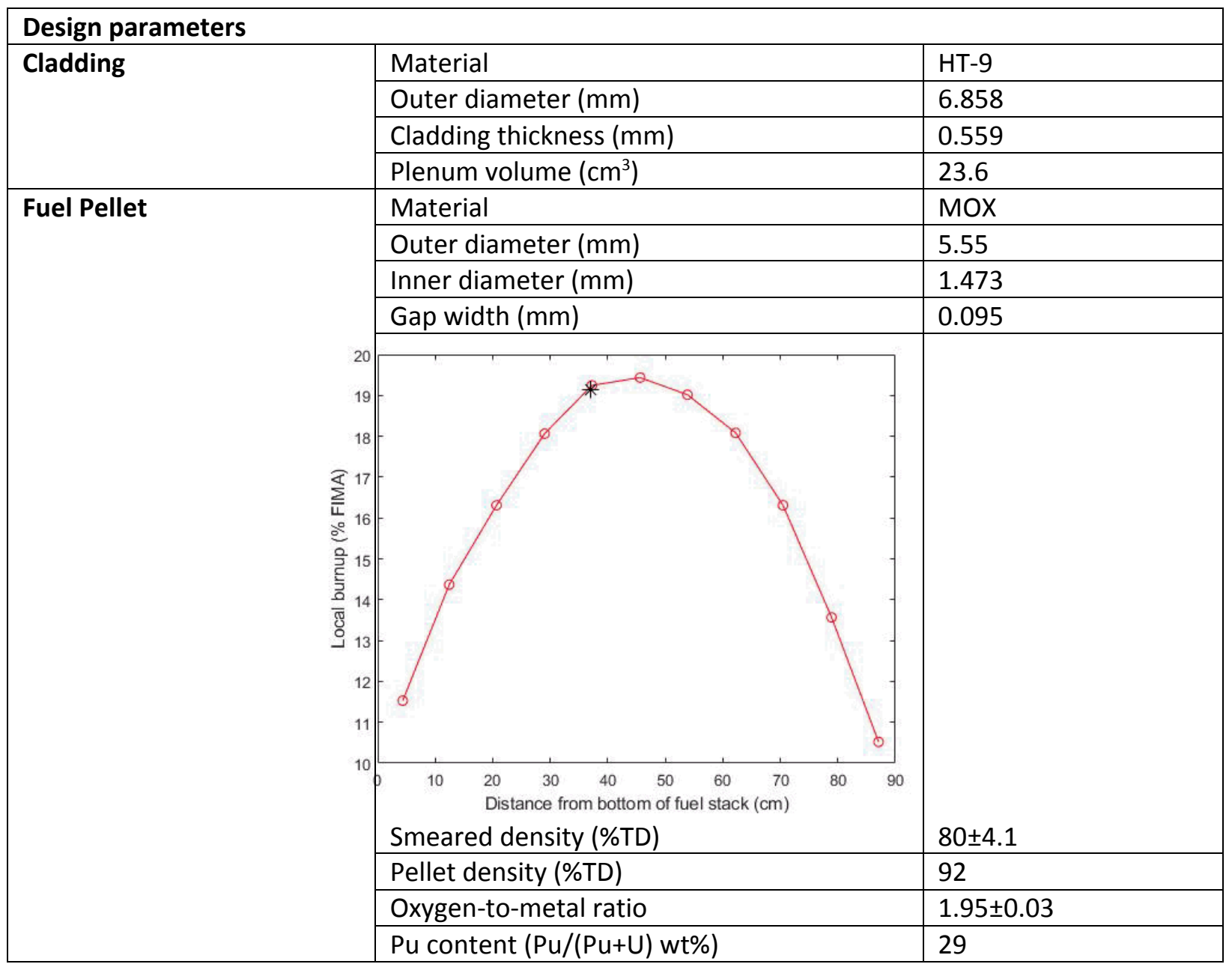

A full cross-section was cut close to power peak node, in the region of highest burnup. Figure 1 reports the sample axial location and the sample burnup as obtained from interpolation of burnup data from [9]. Subsequently, the cross-section was further cut into quarters. The additional sub-sectioning has the multiple advantage of decreasing the overall radioactivity of the sample and reducing the damage to the PFIB detectors. All the cutting and polishing procedures have been carried out using non-water-based compounds in order to avoid washing out of hygroscopic fission products and compounds that accumulate in the fuel-to-cladding gap. The quarter of pellet embedded in the Scanning Electron Microscopy (SEM) stub modified for remote handling with manipulators is shown in Fig. 2.

Figure 1: Axial burnup profile of pin 150074 obtained from analytical chemistry results [9]. The black asterisk marks the axial position of the sample cut for these experiments. 


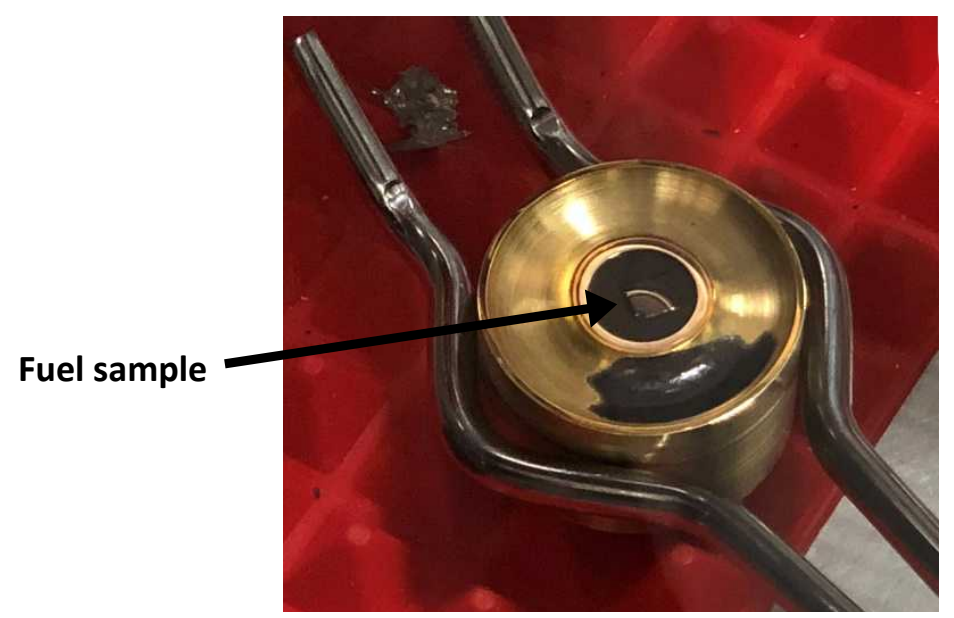

Figure 2: Overview of the quarter pellet embedded in the SEM sample holder.

Four cubes of size approximately $70 \mu \mathrm{m} \times 70 \mu \mathrm{m} \times 30 \mu \mathrm{m}$ depth were cut from the original sample (Fig. 3a) using the FEI Helios PFIB installed at the Irradiated Materials Characterization Laboratory (IMCL). Two cubes were cut close to the central hole of the fuel pellet (cubes 1 and 2), whereas the remaining two (cubes 3 and 4) were cut close to the fuel periphery. The cubes were embedded in pre-cut pockets (Fig. 3b) and welded with Pt in a stainless steel disk of $5 \mathrm{~mm}$ diameter that could be mounted on the mechanical testing equipment.
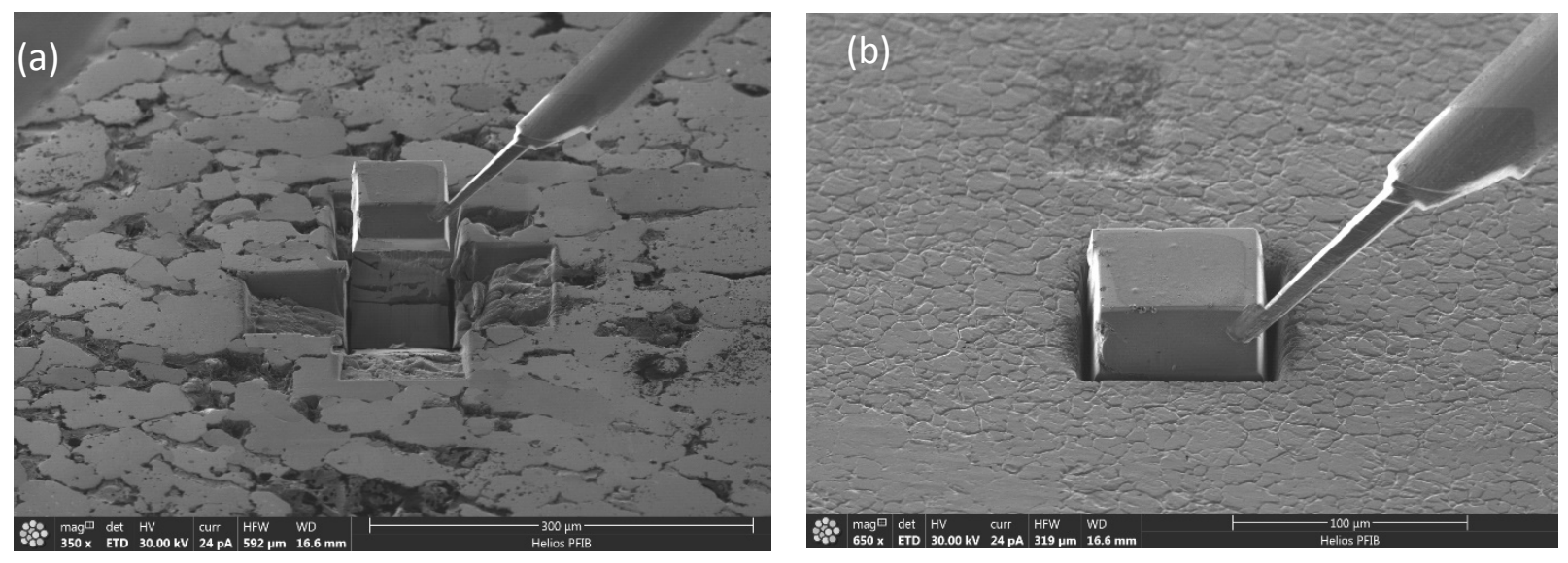

Figure 3: (a) Extraction of cube 2 from the MOX sample. (b) Insertion of cube 2 on the PI 88 sample holder.

Prior to mechanical testing, Electron Backscattered Diffraction (EBSD) data were collected on the MOX cubes using a FEI Quanta FIB to evaluate the grain orientation. Nanoindentation testing was performed at the Electron Microscopy Laboratory (EML) using a Hysitron PI 88 SEM Picolndenter to measure both elastic moduli and hardness. The picoindenter was equipped with a high temperature stage capable of performing indentation up to a temperature of $800^{\circ} \mathrm{C}$. The indenter was first tested on fused silica for calibration. Two tests were conducted at room temperature and one test at $600^{\circ} \mathrm{C}$, all under vacuum. The room-temperature tests were performed in displacement-controlled mode up to a maximum 
displacement of $200 \mathrm{~nm}$. The measured reduced modulus ( $\mathrm{GPa}$ ) was converted to elastic modulus

(GPa) using Equation $1[10]$ :

(1)

Where (GPa) is the indenter Young's modulus, and the sample and indenter Poisson's ratio,

respectively. A Berkovich diamond tip was used for the present tests. Certified Young's modulus and

Poisson's ratio are $1140 \mathrm{GPa}$ and 0.07 , respectively. Regarding the MOX Poisson's ratio, the recommended formula by Martin [11] was used:

Where is the sample deviation from stoichiometry, which was assumed to be the as-fabricated

value.

The hardness value was calculated according to Equation 3: 


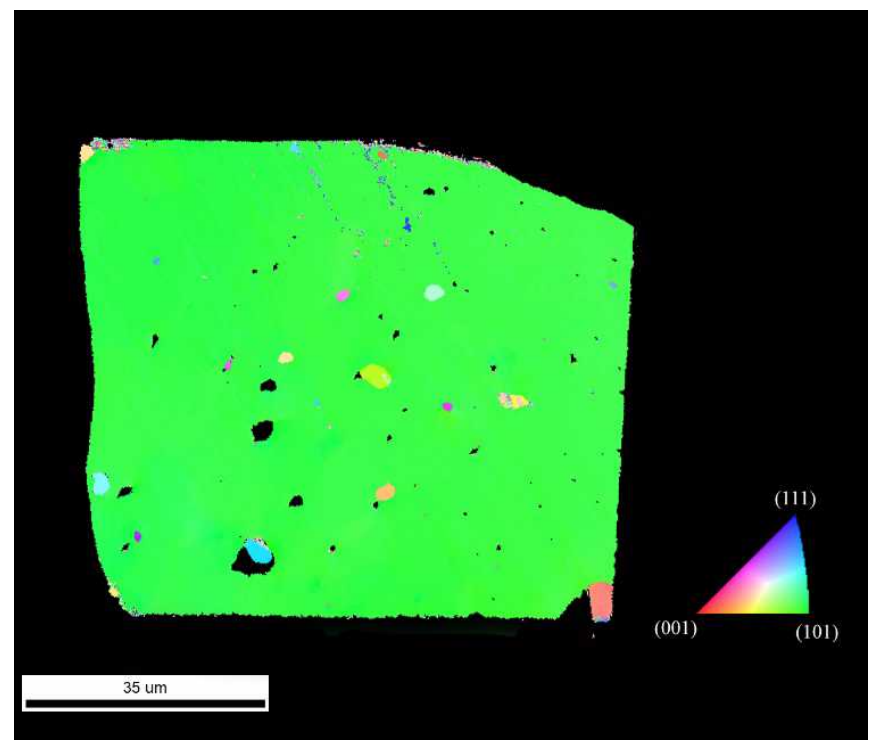

(3)

Where $(\mathrm{Pa})$ is the sample hardness, $(N)$ is the maximum load and $\left(\mathrm{m}^{2}\right)$ is the indentation area.

As the PI 88 stage holds the sample surface parallel to the electron beam, the indentation imprint could be checked only once the PI 88 is removed from the SEM chamber at the end of the measurement campaign. Secondary Electron images were collected with the FEI Quanta following indentation tests to evaluate the imprints.

\section{Results and discussion}

Qualitative inverse pole figures (IPF) were acquired to evaluate the crystallinity of the cubes. An example is shown in Fig. 4. The main phase is composed of a solid solution of $(\mathrm{U}, \mathrm{Pu}) \mathrm{O}_{2}$ oriented in the (101) direction. The small inclusions present with different crystal orientation have either a cubic structure compatible with $(\mathrm{Ba}, \mathrm{Zr}) \mathrm{O}_{3}$ phases or hexagonal structure typical of Mo-Ru-Rh-Tc-Pd noble metal precipitates [12]. Curtaining occurring after removing the platinum protection caused some noise and artifacts in the data close to the cube edges (see Fig. 4). Black regions within in the cube correspond to pores. Cube 1 showed a similar IPF as the one reported in Fig. 4, whereas cube 2 was oriented predominantly in the (111) direction. Cube 4 was polycrystalline, with 3 grains with orientation varying between the (001) direction and the (111) direction. Unfortunately, cube 2 was lost during sample mounting on the PI 88 stage and no indentation data could be collected.

Figure 4: Inverse Pole Figure of cube 3. 
Figure 5 shows the sample holder containing the irradiated MOX cubes mounted on the PI 88 stage. Despite the high burnup and Pu content, the miniaturization of the sample allowed safe handling, following the ALARA principle.
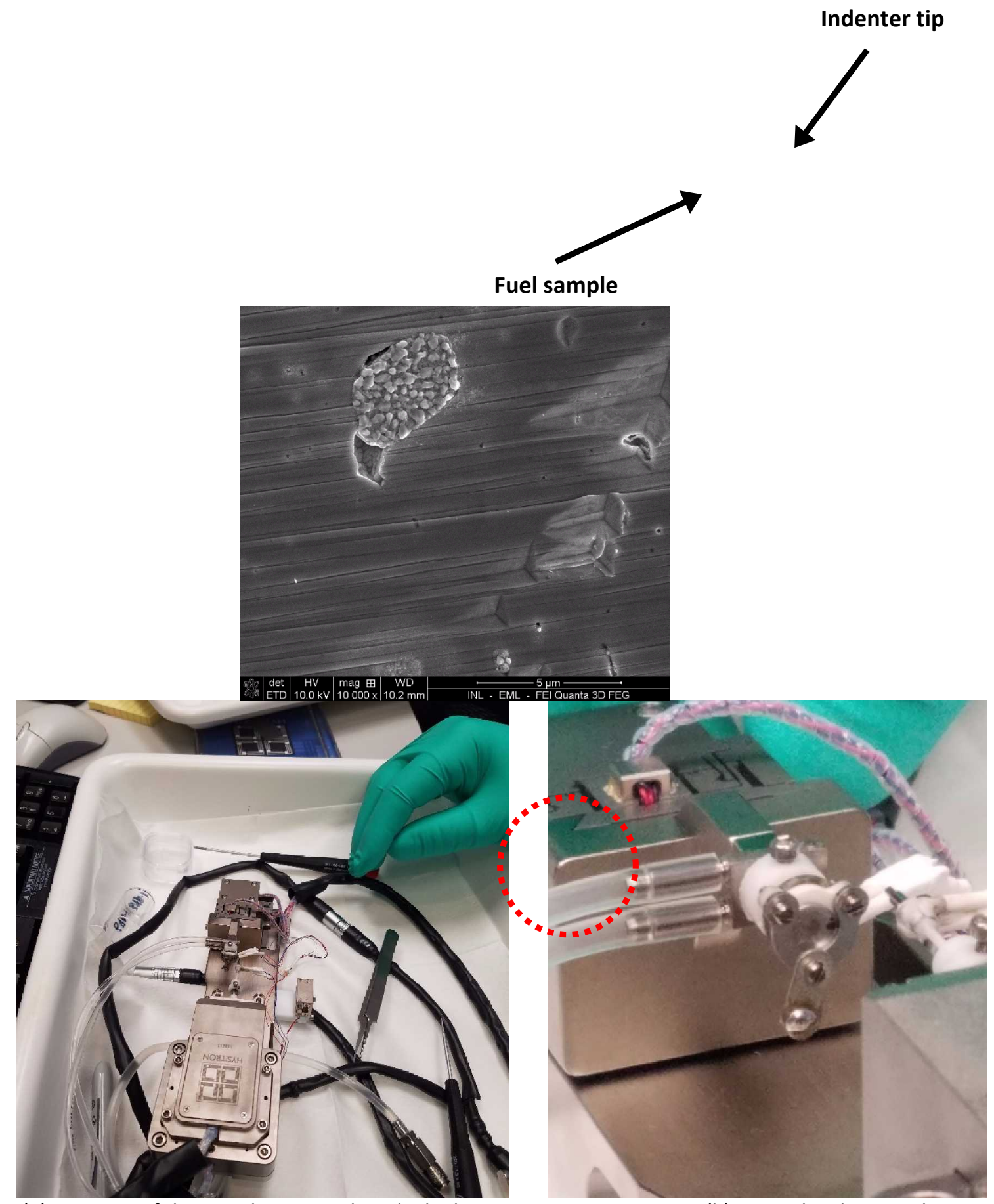

Figure 5: (a) overview of the sample mounted on the high temperature PI 88 stage. (b) Particular showing the 
Figure 6: Post-test SEM image of cube 3 showing the overlapping of indents due to unwanted tip movement during indentation (red circle in the figure).

In addition, post-test inspection of the imprints from the room temperature tests revealed a malfunction of the PI 88 stage motion. The malfunction resulted in a smaller than desired spacing between the indents, with even some overlapping of the indents. None of the measurements performed on cube 4 could be used. Six out of 27 measurements could be used from cube 1 (Fig. 7). Heterogeneity in the Young's modulus and hardness values can be expected due to radiation damage and presence of soft metallic precipitates and porosity, which likely explain the low value of Young's modulus and hardness measured in indent 1.

Figure 7: Young's modulus and hardness values at room temperature on cube 1.

The measured of the sample as local burnup is Based on the that could be present work, it establish result is an limited the literature available or the measurement saturation of the decrease due to products

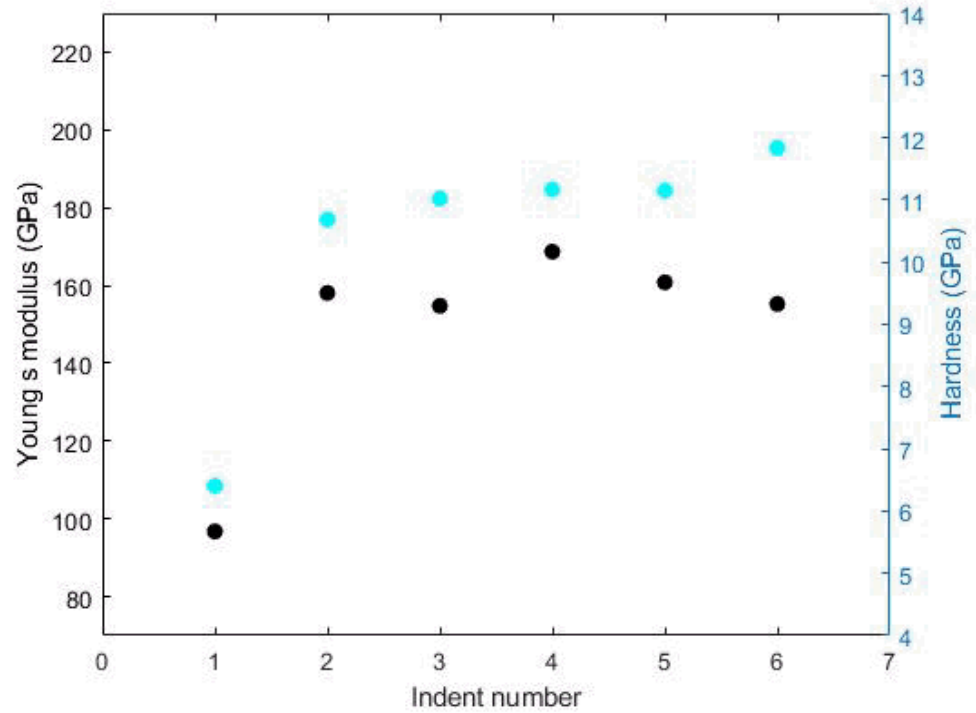

Young's modulus a function of the shown in Fig. 8. single data point collected in the is difficult to whether the outlier with comparability to data so far present suggest that a Young's modulus solid fission accumulation is

occurring at ultra-high burnup. Further work will be performed to strengthen the present observation. 
from this work. Black circles represents published data on irradiated ceramic fuels [3-7].

\section{Conclusions}

The present work represents a pioneering study in the application of advanced techniques to measure micro-mechanical properties of highly radioactive advanced nuclear fuels. First data of Young's modulus and hardness of fast reactor MOX at burnup exceeding $150 \mathrm{GWd} / \mathrm{tHM}$ have been obtained. Despite the challenges encountered in the current work due to equipment malfunction, the experimental approach sought in this work has been proved successful in obtaining new data of high relevance for fuel performance on system with high Pu content and high radiotoxicity with extremely limited exposure to personnel. Further experiments will be conducted in the future based on the present approach, leveraging the experience gained in this project in order to collect enough experimental results for a peer-reviewed publication in the Journal of Nuclear Materials.

\section{Acknowledgements}

This work was supported by the Nuclear Science User Facilities Rapid Turnaround Experiment (Project 18-1452). The authors are indebted to B. Frickey (INL) for sample preparation at HFEF. We would like to acknowledge A. Pomo and M. Taylor (INL) for support during operation in EML.

\section{References}

[1] J. Spino, J. Cobos-Sabate, F. Rousseau, Room-temperature microindentation behaviour of LWRfuels, part 1: fuel microhardness, J. Nucl. Mater. 322 (2003) 204-216. doi:https://doi.org/10.1016/S0022-3115(03)00328-3.

[2] F. Cappia, Investigation of very high burnup UO2 fuels in Light Water Reactors, Technische Universität München, 2017.

[3] F. Cappia, D. Pizzocri, M. Marchetti, A. Schubert, P. Van Uffelen, L. Luzzi, D. Papaioannou, R. Macián-Juan, V.V. Rondinella, Microhardness and Young's modulus of high burn-up UO2 fuel, J. Nucl. Mater. 479 (2016). doi:10.1016/j.jnucmat.2016.07.015.

[4] D. Laux, D. Baron, G. Despaux, A.I. Kellerbauer, M. Kinoshita, Determination of high burn-up nuclear fuel elastic properties with acoustic microscopy, J. Nucl. Mater. 420 (2012) 94-100. doi:https://doi.org/10.1016/j.jnucmat.2011.09.010.

[5] M. Marchetti, D. Laux, L. Fongaro, T. Wiss, P. Van Uffelen, G. Despaux, V. V Rondinella, Physical and mechanical characterization of irradiated uranium dioxide with a broad burnup range and different dopants using acoustic microscopy, J. Nucl. Mater. 494 (2017) 322-329. doi:https://doi.org/10.1016/j.jnucmat.2017.07.041.

[6] K.A. Terrani, M. Balooch, J.R. Burns, Q.B. Smith, Young's modulus evaluation of high burnup structure in UO2 with nanometer resolution, J. Nucl. Mater. 508 (2018) 33-39. doi:https://doi.org/10.1016/j.jnucmat.2018.04.004.

[7] D. Laux, W. de Weerd, D. Papaioannou, S. Kitajima, V. V Rondinella, G. Despaux, Scanning 
acoustic microscope for mechanical characterization and density estimation of irradiated nuclear fuel, Prog. Nucl. Energy. 72 (2014) 63-66. doi:https://doi.org/10.1016/j.pnucene.2013.07.018.

[8] J.A. Lancaster, N.J. Graves, A.E. Bridges, Irradiation history for advanced oxide fuel assembly ACO3, Richland, Washington, 1992.

[9] M. Teague, Post Irradiation Examination of Legacy FFTF Oxide Fuel, 2012.

[10] W.C. Oliver, G.M. Pharr, Nanoindentation in materials research: Past, present, and future, MRS Bull. 35 (2010) 897-907. doi:DOI: 10.1557/mrs2010.717.

[11] D.G. Martin, The elstic constants of polycrystalline UO2 and (U,Pu) mixed oxides: a review and recommendations, High Temp. - High Press. 21 (1989) 13-24.

[12] H. Kleykamp, The Chemical State of Fission Products in Oxide Fuels at Different Stages of the Nuclear Fuel Cycle, Nucl. Technol. 80 (1988) 412-422. doi:10.13182/NT88-A34065. 